\title{
NTRK2 Gene Alteration Positive
}

National Cancer Institute

\section{Source}

National Cancer Institute. NTRK2 Gene Alteration Positive. NCI Thesaurus. Code C134453.

A finding indicating that mutation, overexpression or rearrangement of the NTRK2 gene has been detected in a sample. 